\title{
CONSTRAINT-BASED EXCLUSION OF LIMB POSES FOR RECONSTRUCTING THEROPOD DINOSAUR LOCOMOTION
}

\author{
STEPHEN M. GATESY, ${ }^{* 1}$ MARTIN BÄKER, ${ }^{2}$ and JOHN R. HUTCHINSON ${ }^{3}$ \\ ${ }^{1}$ Department of Ecology and Evolutionary Biology, Brown University, Providence, Rhode Island \\ 02912 U.S.A., stephen_gatesy@brown.edu; \\ ${ }^{2}$ Institut für Werkstoffe, Technische Universität Braunschweig, Langer Kamp 8, 38106 \\ Braunschweig, Germany, martin.baeker@tu-bs.de; \\ ${ }^{3}$ Structure and Motion Laboratory, The Royal Veterinary College, University of London, \\ Hatfield, Hertfordshire AL9 7TA, United Kingdom, jrhutch@RVC.AC.UK
}

\begin{abstract}
Plausible poses were identified for the hind limb of Tyrannosaurus rex and three other non-avian theropod dinosaurs at mid-stance of locomotion using constraint-based exclusion. This new method, validated by analysis of two species of birds, involves applying demonstrably realistic geometric and kinetic (force-based) constraints from extant animals to exclude, rather than include, potential poses. Starting with a "configuration space" of millions of candidate poses, we used a step-wise series of criteria to constrict the volume to a small subset of solutions, which can serve as starting points for reconstructing complete stride cycles. It was found that the maximum relative mid-stance limb force, as well as the relative number of configurations at lower forces, decreased with increasing body size. Constraint-based exclusion restricted Tyrannosaurus to a narrow region of neither very columnar nor very flexed poses that may have allowed relatively slow running, but no reasonable combinations of input parameters and pose produce forces large enough for high speeds. This analysis shows that skeletal information alone has limited value for discerning mid-stance poses. Despite additional assumptions, unpreserved parameters such as masses, forces, and moments are required to study a fossil as a functioning animal, rather than as a moving set of bones. Constraint-based exclusion is a transparent, reproducible framework for evaluating functional hypotheses in dinosaurs and other taxa.
\end{abstract}

\section{INTRODUCTION}

Paleobiologists strive to reconstruct movement in extinct animals to help bring their fossilized remains "back to life" in ways that static anatomy cannot. Reconstructed motions of individual taxa are often critical for making higher-level inferences about the patterns of functional, behavioral, and ecological change through time, as well as for fully documenting the diversity of adaptations in the history of life. Major evolutionary transitions such as the origin of limbs and terrestriality, conversions from sprawling to erect posture, shifts from quadrupedalism to bipedalism and back, returns to an aquatic habitat, and the advent of powered flight primarily entailed transformations in how vertebrates transmitted motive forces to their environment. In the case of theropod dinosaurs, changes in primitive hind limb posture and gait have been proposed along the line to extant birds (e.g., Gatesy, 1990) and at extremely large body size (e.g., Hutchinson and Garcia, 2002). To test these hypotheses, we require improved methods for quantitatively reconstructing locomotor poses in extinct taxa.

A key problem is that studies of motion from fossil evidence confront daunting uncertainties (reviewed in Hutchinson and Gatesy, 2006). Vertebrate joints are mobile enough to permit an animal's full movement repertoire, but such total ranges of motion do not unambiguously delineate actual excursions during any single behavior. Moreover, appendages such as limbs, fins, necks, and tails have tremendous redundancy-what engineers refer to as excess degrees of freedom-that requires knowledge of coordination among joints/segments. Timing presents yet

\footnotetext{
*Corresponding author.
}

another conundrum because velocities, durations, and frequencies are not preservable data. These spatial and temporal unknowns evoke a larger scientific question: how can we maximize the rigor of reconstructions while minimizing speculation?

In this article, we address the question of reanimating extinct taxa using the hind limb of Tyrannosaurus rex, a case study for which stance, gait, and top speed are controversial (Osborn, 1916; Lambe, 1917; Newman, 1970; Tarsitano, 1983; Bakker, 1986; Paul, 1988, 1998; Horner and Lessem, 1993; Farlow et al., 1995; Hutchinson and Garcia, 2002; Hutchinson, 2004a, 2004b; Hutchinson et al., 2005; Sellers and Manning, 2007). There are no $6000+\mathrm{kg}$ bipeds alive today; how could one have stood and moved, and how did its locomotor system evolve? Herein, we specifically focus on the problem of spatial uncertainty created by joint mobility and excess degrees of freedom. Our goal is to identify, based on our understanding of extant bipeds, biomechanically justifiable hind limb poses that Tyrannosaurus could have assumed when moving at constant speeds, and compare these poses with those of other extant and extinct theropods. Rather than attempting to reconstruct entire strides (e.g., Sellers and Manning, 2007), we start more modestly by searching for viable limb postures used near the middle of the stance (foot contact) phase of steady locomotion (see below). By focusing on this single instant, we can analyze the limb when the musculoskeletal system is highly stressed and postpone dealing with the issue of temporal uncertainty. Our aim is to use mid-stance solutions as anchor points for future studies reconstructing complete stride cycles in theropod dinosaurs, to better reconstruct the evolution of stance and gait.

We begin by creating a "configuration space" of potential hind limb poses, casting a wide net in order to avoid missing possible solutions. We then exclude non-viable poses using a 
step-wise series of constraints to carve down the volume, like a block of stone, to reveal a small subset of solutions. "Hard," geometric criteria are treated first to establish the extent to which mid-stance limb position can be discerned from skeletal evidence alone. We then reconstruct relevant soft tissues to invoke kinetic (force-based) constraints, validating our specific assumptions with data from living bipeds. Viable running poses for Tyrannosaurus are compared to results from a living ostrich and emu as well as other theropods. We then use our sample to examine how maximal limb forces and allowable limb configurations scale with body size. Strengths and weaknesses of constraint-based exclusion and future directions are discussed relative to other methods.

\section{MATERIALS AND METHODS}

Hind limb data from seven species (four extinct and three extant) were used in this study. We present the largest, Tyrannosaurus rex, as an example to describe our methodology. Threedimensional polygonal models (Fig. 1A; Hutchinson et al., 2005) of the pelvis and hind limb of T. rex (MOR 555, Museum of the Rockies, Boseman, Montana, USA) were imported into Maya 8.5 (Autodesk, Inc.) and SIMM (Musculographics, Inc.). Motion was restricted to sagittal flexion/extension; the pelvis was oriented horizontally (Newman, 1970; Paul, 1988). The centroid of the femoral head (hip), midpoint between the centroids of the lateral and medial femoral condyles (knee), centroid of the astagalus/ calcaneum (ankle), and centroid of the third metatarsal condyle (metatarsophalangeal) were designated as joint centers. The proximal phalanx of the third toe was dorsiflexed relative to the distal, horizontal phalanges based on dinosaur footprints (e.g., Gatesy et al., 1999; Farlow et al., 2000) and avian kinematics (e.g., Gatesy, 1999, unpublished data; Middleton, 2003). Soft tissue digital pads were estimated (considering the anatomy of extant birds) to extend below and behind the phalanges (Table).

By fixing the position of the phalanges and the orientation of the pelvis, the hind limb's pose can be described by any three of the four main joint angles (Fig. 1B). A "configuration space"
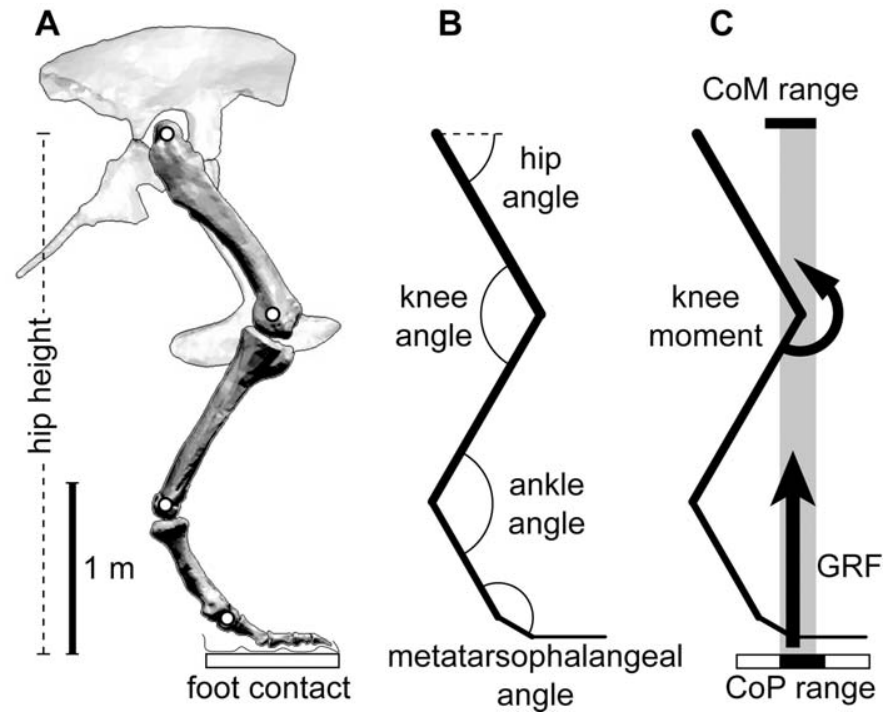

FIGURE 1. Tyrannosaurus hind limb. A, right lateral view of the limb and pelvis model with estimated joint centers (circles) and foot contact (white bar); B, joint angles used to describe limb pose and ranges of motion; C, unpreserved kinetic variables used in the analysis. At midstance, a vertical GRF (large arrow) can only exist if there is overlap (grey box) between the possible anteroposterior locations of the CoM (upper black bar) and CoP (lower black bar). Limb collapse is prevented by extensor muscle moments about joints such as the knee (small arrow). of possible poses was visualized by plotting combinations of hip, knee, and ankle angle as a 3-D graph in Matlab 7.0 (The Mathworks, Inc.) and Maya. Hip angle was varied between $0^{\circ}$ (femur protracted to horizontal) and $180^{\circ}$ (femur retracted to horizontal). Both knee and ankle angles ranged from $0^{\circ}$ (fully flexed) to $180^{\circ}$ (fully extended). The metatarsophalangeal angle was allowed to vary freely. Angles were analyzed in $1^{\circ}$ increments along each axis, yielding nearly six million $\left(181^{\circ} \times 181^{\circ} \times 181^{\circ}=\right.$ $5,929,741)$ potential poses in the intentionally over-sized volume (Fig. 2A)

\section{Mid-Stance}

During the stance phase of locomotion, the foot transmits force to the ground, which pushes back with an equal but opposite ground reaction force (GRF; see Farley and Ferris, 1998 for a review). In steady-speed running, the GRF is maximal about halfway through foot contact. We used this ubiquitous pattern to specifically define mid-stance as the instant when the fore-aft component of force is zero, at which time the GRF projects vertically upward (Fig. 1C) from the foot's center of pressure (CoP). As living bipeds move, the GRF closely targets the body's center of mass (CoM) (Alexander, 2004; Herr and Popovic, 2008), so we assumed that in Tyrannosaurus the whole body $\mathrm{CoM}$ was directly over the $\mathrm{CoP}$ at mid-stance. Unlike some skeletal parameters, variables such as the locations of the CoM and $\mathrm{CoP}$ and the magnitude of the GRF remain unknown. For each pose in the configuration space, we evaluated 16 potential trunk CoM positions between $0.4 \mathrm{~m}$ and $0.7 \mathrm{~m}$ anterior to the hip joint (Hutchinson et al., 2007) in $0.02 \mathrm{~m}$ increments (the ventral distance of the CoM from the hip was not required for the analysis). The CoP was confined to the middle third of the contact length of the foot (Table; Fig. 1C), based on force and pressure plate data from extant bipeds (Roberts and Scales, 2002; Hallemans et al., 2003; Middleton, 2003; Vereecke et al. 2003, 2005; McGowan et al., 2005).

\section{Constraints}

Geometric constraints included ranges of joint motion and ground penetration. A logical starting point was to exclude regions of configuration space outside the limits of flexion/extension estimated from articular morphology (Table). We then disqualified poses in which the knee, ankle, or pubis fell below the ground surface, assuming reasonable bone and soft tissue radii around each joint center (Table).

Five criteria were used as kinetic constraints: vertical GRF, correct moment sign, relative moment magnitudes, maximum hip height, and absolute muscle moment magnitudes. These are ordered from less to more specific, adding additional assumptions in order to increase resolution. Our first "soft" constraint was a vertical GRF, which excluded any configuration that did not have at least one potential whole body CoM position over the middle third of foot contact. Second, we barred mid-stance poses in which one or more joints had net muscle moments of the wrong sign. In living bipeds, muscles prevent limb collapse by producing net extensor (anti-gravity) moments about the hip, knee, and ankle at mid-stance (Biewener et al., 1981; Roberts, 2001; Günther and Blickhan, 2002; Rubenson et al., 2003; McGowan et al., 2005; Main and Biewener, 2007). Using a free-body-diagram approach (Hutchinson and Garcia, 2002; Hutchinson, 2004a, 2004b), we calculated moments and excluded poses entailing net flexor muscle moments about any of these joints. Inertial moments, which would be small at mid-stance (Biewener et al., 2004) and depend on knowledge of angular acceleration, were neglected.

Third, we evaluated relative moment magnitudes. As in living tetrapods, Tyrannosaurus had tendons from long digital flexor musculature passing behind the ankle and beneath the 
TABLE. Geometric and kinetic variables for mid-stance pose evaluation.

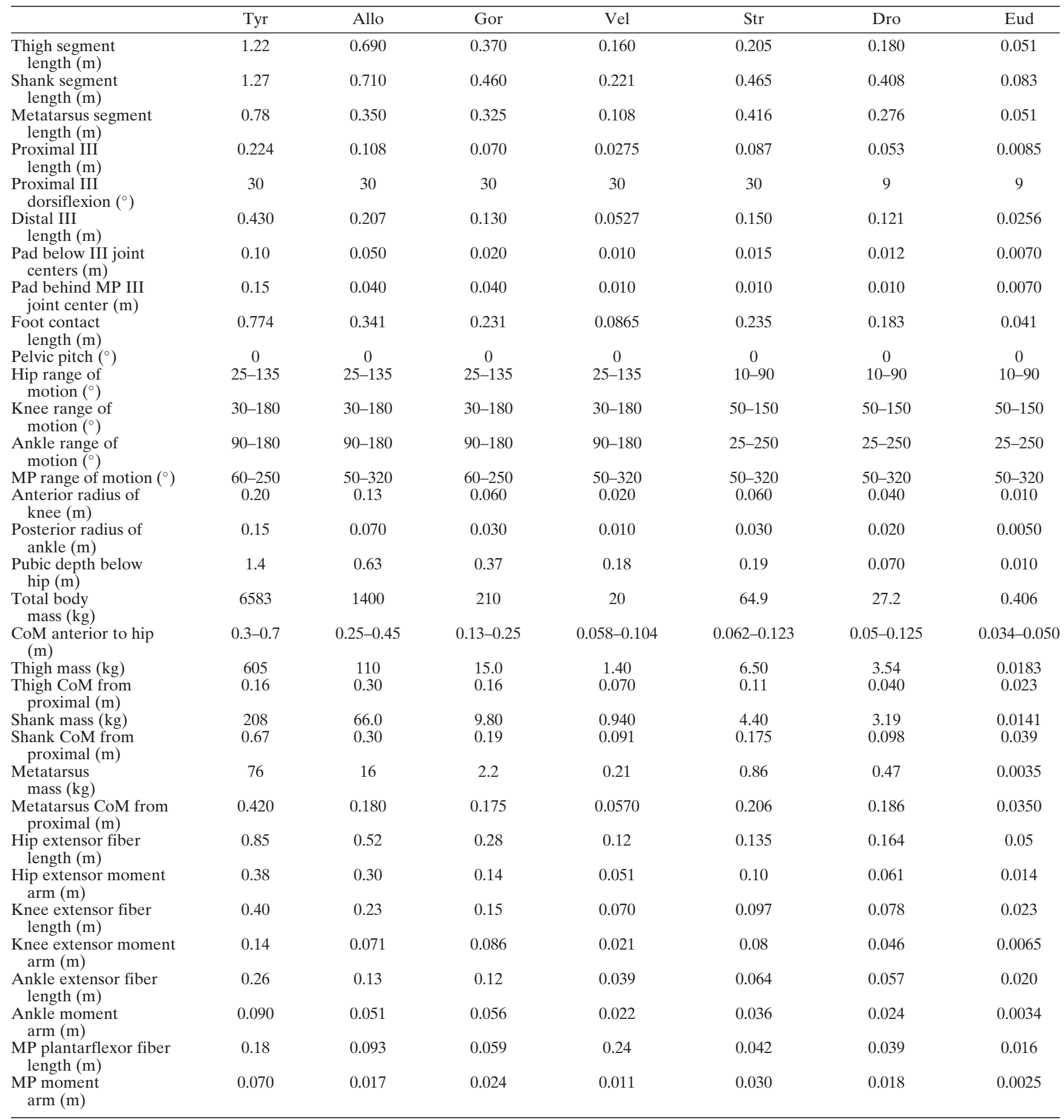

Abbreviations: Allo, Allosaurus; Dro, Dromaius; Eud, Eudromia; Gor, Gorgosaurus (juvenile); Str, Struthio; Tyr, Tyrannosaurus; Vel, Velociraptor.

metatarsophalangeal joints to help prevent limb collapse. The ankle moment should be greater than the metatarsophalangeal moment (Alexander et al., 1979), due to both its larger average moment arm (Table; Hutchinson et al., 2005) and force from additional extensor muscles, such as the gastrocnemius. Fourth, we set a maximum hip height threshold. During running, a hind limb contacts the ground and shortens through early stance, storing elastic strain energy and bringing the hip to its lowest point near mid-stance (Cavagna, Heglund, and Taylor, 1977; McMahon and Cheng, 1990). If the hip is too high at mid-stance, the leg spring will not be adequately compressed. Moreover, a limb that is too high at touchdown cannot reach forward far enough to produce a step of sufficient length. Determining a precise cutoff for maximum hip height using validated principles 
from extant taxa is problematic, so we chose a very conservative value of $95 \%$ crouch to $3.31 \mathrm{~m}$.

Finally, absolute muscle moment magnitudes were computed to exclude any pose that requires an unreasonably large mass of extensor musculature. The peak vertical GRF of Tyrannosaurus is not known, so we began with a value of one body weight $(1 \mathrm{BW})$ and tested larger magnitudes to determine a maximum GRF beyond which no poses were deemed viable. Following Roberts (2001), the maximum isometric extensor muscle moment for each joint was conservatively estimated as the force produced by complete activation of a volume of muscle $\left(5 \%\right.$ of body mass; density of $1060 \mathrm{~kg} / \mathrm{m}^{3}$, Mendez and Keys, 1960) with a mean fascicle length (Table; Hutchinson et al., 2004a) and typical isometric stress $\left(300 \mathrm{kNm}^{-2}\right.$, Medler, 2002) multiplied by a fixed moment arm (Table; based on mean values from Hutchinson et al., 2005). Our assumption of isometry is in general agreement with data from living bipeds, in which extensor muscles produce force with very little length change during the stance phase of level, steady-speed locomotion. Tendons recoil elastically to provide power (e.g., Roberts et al., 1997; Roberts, 2002), but excluding these passive components from our model should not skew our estimates of muscle required.

\section{Validation}

We validated the method by searching for viable mid-stance poses in two large ratite birds, the ostrich (Struthio camelus) and the emu (Dromaius novaehollandiae). Body masses, skeletal lengths, segment masses, segment centers of mass, extensor muscle fiber lengths, and extensor muscle moment arms were from two previously described individuals (Table; Hutchinson, 2004a). The trunk CoM was varied over a range (Table) in $0.005 \mathrm{~m}$ increments. As with Tyrannosaurus, we assumed 5\% of body mass in extensor musculature at each joint and used identical assumptions about muscle density, isometric muscle stress, inertia, and CoP. Joint ranges of motion were estimated from skeletal elements only. Maximum hip height was set at $95 \%$ of the tallest pose allowed by joint ranges of motion. Starting with a full configuration space of $8,223,011$ poses $\left(0-180^{\circ}\right.$ in $1^{\circ}$ increments for hip and knee, $0-250^{\circ}$ for ankle), we applied the same constraints in the same sequence. The remaining volumes were compared to actual mid-stance poses used by two ostriches running over a forceplate at about $3.3 \mathrm{~ms}^{-1}$ (Jindrich et al., 2007; Rubenson et al., 2007) and a representative pose just prior to mid-stance from running emus (Main and Biewener, 2007:fig. 2C).

\section{Comparative Analysis}

Data for individual specimens of other species (Table) were taken from Hutchinson (2004a, 2004b) to study the effect of scaling: the extinct theropods Allosaurus, Gorgosaurus (juvenile), and Velociraptor, and an extant Elegant-crested tinamou (Eudromia). We could not use the tinamou for our validation because reliable experimental data on mid-stance poses are unavailable. To compare results, we plotted the relative number of configurations for each of the species studied for different values of the peak relative mid-stance GRF. The number of configurations was normalized for each species by dividing it by the number of configurations when all constraints except for the absolute muscle moment magnitudes were applied. Normalization at least partially accounts for differences in joint ranges of motion, uncertainty in CoM position, and limb proportions. We also searched for the optimum configuration for generating maximal possible vertical GRF in each taxon. Finally, the configuration with the lowest hip height capable of producing a GRF of 1.5 BW was also compared among all species. Relative minimum crouch was calculated as the lowest pose corresponding to slow running as a percentage of the tallest hip height permitted by range of motion constraints.

\section{RESULTS}

We first present results for Tyrannosaurus in full as an example of the method, followed by summaries of the validation and scaling analyses.

\section{Tyrannosaurus Mid-Stance Poses}

Starting with almost six million candidates (Fig. 2A), our estimates of maximum hip, knee, and ankle flexion/extension remove any poses falling outside a rectangular block of configuration space. Limiting metatarsophalangeal angle slices off two corners of this block. Together, ranges of motion for these four joints eliminate more than $80 \%$ of the poses, but a volume of 1,120,886 alternatives (18.9\% of total; Fig. 2B) remains. Excluding poses in which the knee, ankle, or pubis fall below the ground surface leaves 970,463 solutions $(16.4 \%$; Fig. 2C).

Enforcing a vertical GRF (CoM range overlapping $\mathrm{CoP}$ range) severely restricts the horizontal offset between hip and toes. If exact locations were known, viable poses would be restricted to a two-dimensional surface in configuration space. Instead, our ranges of uncertainty limit mid-stance solutions to a slab-like volume composed of 111,613 solutions $(1.88 \%$; Fig. 2D). The correct moment sign constraint further limits the volume to 70,073 solutions ( $1.18 \%$; Fig. $2 \mathrm{E})$, primarily by removing poses with hip angles large enough to place the knee axis behind the GRF. Constraining the ankle moment to be greater than or equal to the metatarsophalangeal moment eliminates poses in which the metatarsus is rotated forward past vertical, leaving 51,723 solutions $(0.87 \%$; Fig. 2 F). The maximum hip height constraint crops off alternatives taller than $3.31 \mathrm{~m}$, yielding 50,340 poses $(0.85 \%$; Fig. $2 \mathrm{G})$.

Given our assumptions about muscle size, architecture, and force generation, 27,210 poses $(0.46 \%$; Fig. $2 \mathrm{H})$ are able to sustain a GRF of $1 \mathrm{BW}$ as required for standing statically on one leg. In extant animals, peak GRF magnitude positively correlates with speed (Alexander et al., 1979; McMahon and Cheng, 1990; Munro et al., 1987). Running may be possible with a mid-stance GRF of as low as $1.5 \mathrm{BW}$, but living bipeds typically generate at least $2 \mathrm{BW}$, reaching 2.5-4 BW at higher speeds (summarized in Hutchinson, 2004a). Only 2,391 solutions (0.04\%; Fig. 2I) can support $1.5 \mathrm{BW}$; none can accomplish a mid-stance GRF greater than $1.87 \mathrm{BW}$. We therefore conclude that for an adult Tyrannosaurus the maximum, steady-speed GRF must have been less than $2 \mathrm{BW}$, consistent with relatively slow running at best.

\section{Ratite Mid-Stance Poses}

The ostrich's and emu's patterns of incremental reduction in configuration space volume are quite similar to that of Tyrannosaurus for all constraints except absolute moment magnitude. For Struthio, joint ranges of motion eliminate most of the original eight million poses, leaving 1,511,872 solutions (18.4\%). Preventing ground penetration retains 1,210,092 possibilities (14.7\%). As with Tyrannosaurus, requiring at least some overlap between CoM and CoP ranges is important, reducing the volume to 153,837 poses $(1.87 \%)$. Constraining correct moment sign (90,667 poses: $1.10 \%)$, relative moment magnitude $(74,591$ poses: $0.91 \%$ ), and maximum hip height leaves a sliver of 71,517 potential poses $(0.87 \%)$. In contrast to Tyrannosaurus, which can only achieve a GRF of $1.5 \mathrm{BW}$ with 2,391 poses, all 71,517 poses that pass the maximum hip height constraint can be used by the ostrich for slow running (Fig. 3A). We obtained comparable results for the emu. 

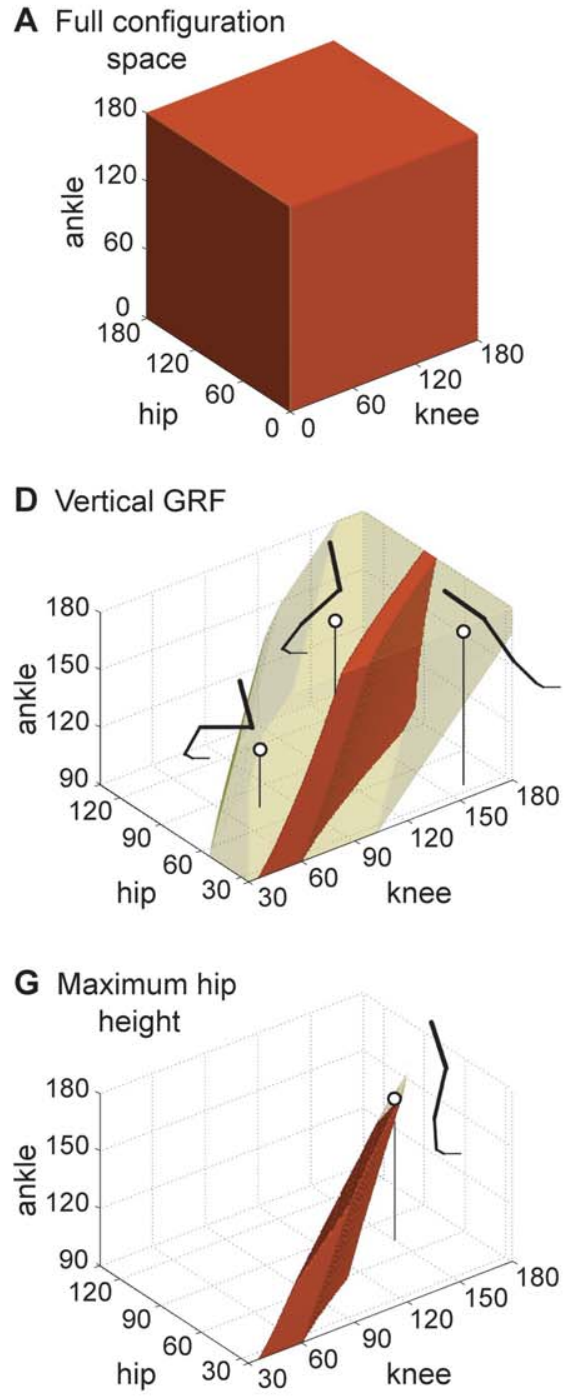

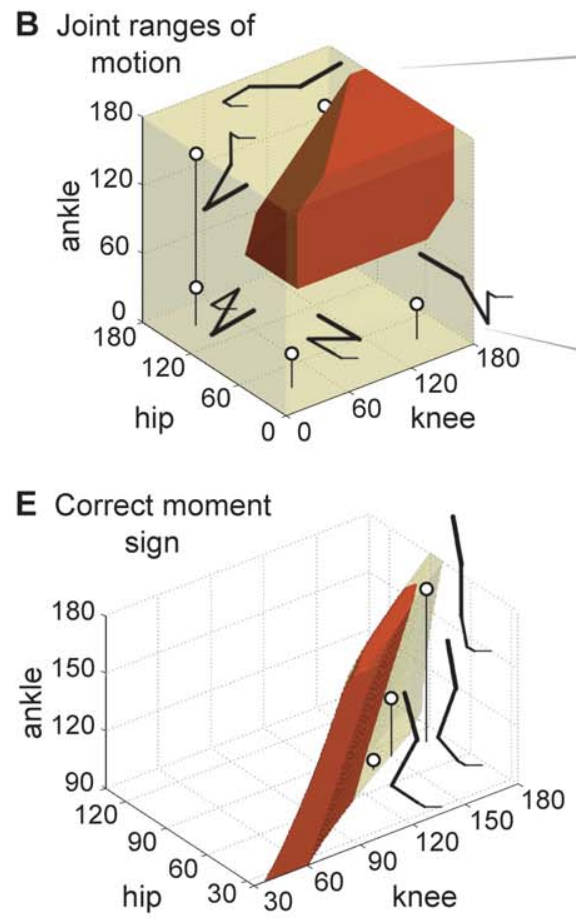

C Ground penetration
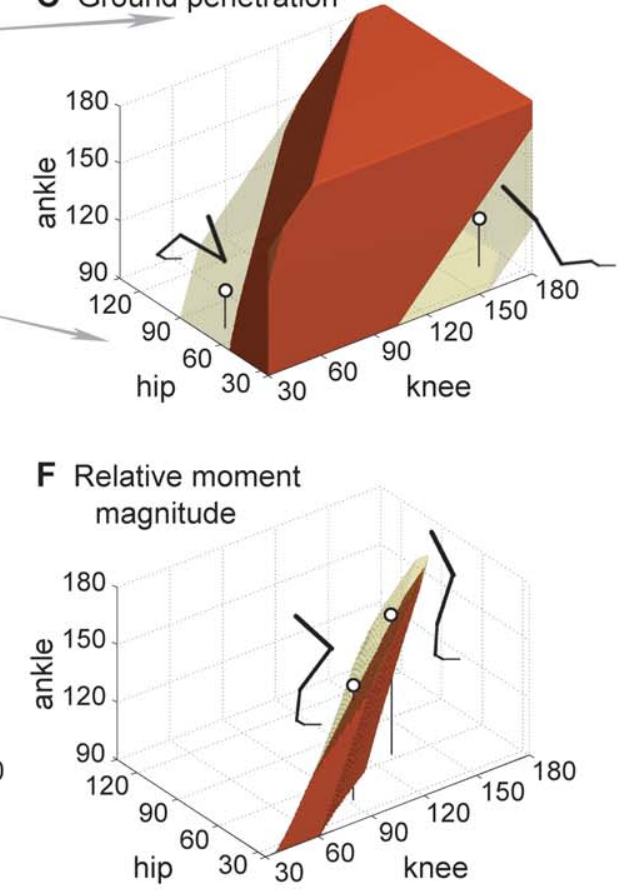

$H$ GRF $=1 B W$

$\mathrm{I} \mathrm{GRF}=1.5 \mathrm{BW}$
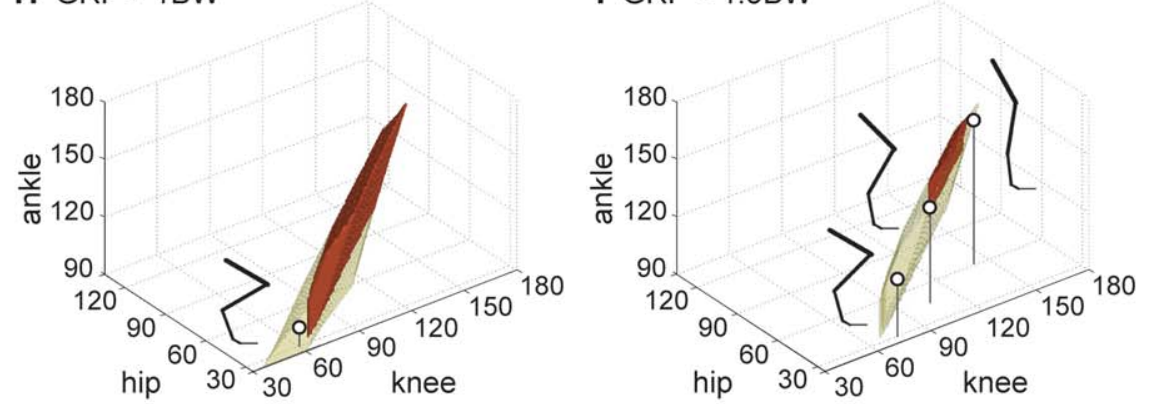

FIGURE 2. Constraint-based exclusion of mid-stance poses for Tyrannosaurus showing viable mid-stance poses (solid red), excluded volumes (transparent), and sample excluded poses (white circles and stick figures). Note change in axes between B and C.

Both quantified mid-stance poses published for ostriches (pose 1, Jindrich et al., 2007: hip 48 ${ }^{\circ}$, knee $109^{\circ}$, ankle $142^{\circ}$; pose 2, Rubenson et al., 2007: hip $33^{\circ}$, knee $103^{\circ}$, ankle $159^{\circ}$ ) fall within the predicted volume (Fig. $3 \mathrm{~A}, \mathrm{~B}$ ). Pose variation is expected given the difficulty of measuring femoral orientation, intraspecific disparity, and methodological differences, as well as slight deviations in GRF direction. The emu pose shown by Main and Biewener (2007:fig. 2C) represents an instant near mid-stance just prior to a fully vertical GRF, yet given our uncertainty about the location of the CoM, still falls within our volume of running poses with a GRF of at least 1.5 BW.

\section{Comparative Analysis}

Our results for the other avian and non-avian species are similar for constraints prior to absolute muscle moment magnitude. Thereafter, the relative number of permissible configurations reveals obvious patterns as relative GRF increases (Fig. 4A). Non-avian theropods show a clear scaling trend, with larger animals descending to their maximum GRF more quickly. The three bird plots exhibit shallower slopes; ostriches and emus

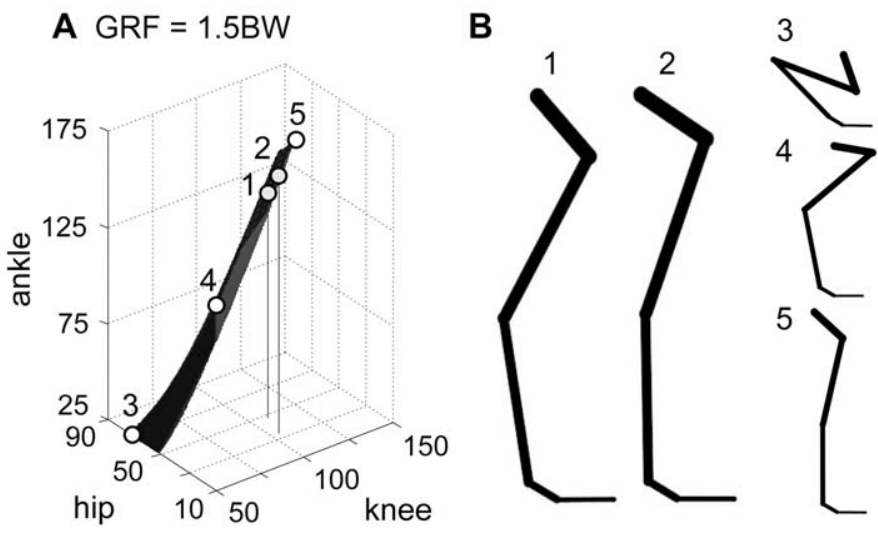

FIGURE 3. Constraint-based exclusion of mid-stance poses for Struthio. A, ostriches can produce a GRF of $1.5 \mathrm{BW}$ needed for running using a large number of alternatives (grey volume); $\mathbf{B}$, five potential poses include two (1 and 2, circles in $\mathbf{A}$ ) actually measured in running ostriches. 

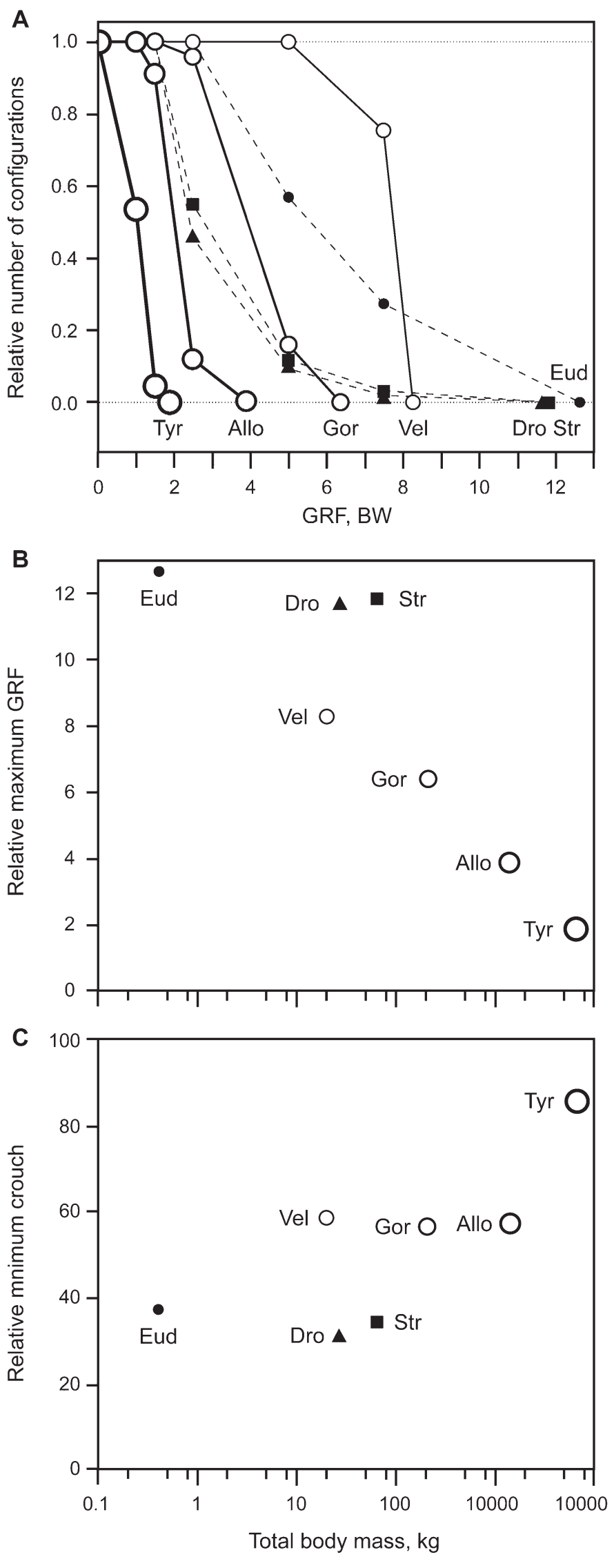

are quite similar and decline before the smaller tinamou (Fig. 4A). Theoretical maximum vertical GRFs show a strong effect of size (Fig. 4B). As expected, maximal GRF declines steeply with increasing body mass, although ratite birds stand out as having maximal GRF capacity above the non-avian theropod trend. Relative minimum crouch (Fig. 4C) for slow running (GRF of $1.5 \mathrm{BW}$ ) is relatively constant in the three birds and for three of the non-avian theropods. The similarity among minima for each group may have little significance because poses with less crouch could have been used to run. Only the large-bodied Tyrannosaurus is tightly constrained by a minimum hip height ( $85 \%$ crouch) approaching its maximum hip height (95\%) during running.

\section{DISCUSSION}

In this paper, we offer a strategy in which a series of constraints is used to exclude, rather than include, possible limb configurations for the hind limb of Tyrannosaurus and other extinct theropods at one instant during the stride cycle. Our goal was to formalize the exclusion process by using demonstrable biomechanical constraints, eschewing intuition and loose rules of thumb. Instead of restricting analysis to several candidate poses for Tyrannosaurus (Hutchinson and Garcia, 2002; Hutchinson, 2004b), a broad space of almost 95 million combinations was searched (six million poses each evaluated at $16 \mathrm{CoM}$ positions). From this vast pool, sequentially imposing geometric and kinetic filters (Fig. 2) ultimately carved the full space down to 2,391 poses consistent with slow running.

Geometric constraints most directly preserved in skeletal morphology proved essential at the most basic level (segment lengths, limb motion primarily parasagittal), but lacked power to resolve mid-stance poses in any detail. Although functional analyses often estimate joint ranges of motion from articular geometry (Gishlick, 2001; Carpenter, 2002; Senter, 2005), results from pose exclusion are sobering. For example, even when Tyrannosaurus is restricted to flexion/extension, major joint excursion limits (Fig. 2B) and the ground penetration constraint (Fig. 2C) still permit almost a million alternatives. The final solution set (Fig. 2I) has no borders delineated by geometric constraints. Our estimates of mobility may have been too liberal, but these joints are likely not near their limits at mid-stance. Geometric constraints could be more important at other times within the stride and in other behaviors (e.g., lying down, feeding, etc.) when muscle forces are lower, limb lengths are longer/ shorter, or ground penetration is a greater impediment.

The contribution of imposing kinetic constraints is obvious from Figure 2. Even with our uncertainty about the locations of the CoM and CoP, the vertical GRF constraint eliminated seven of every eight geometrically permissible poses. The power of even this simple kinetic relationship underscores that the most promising way forward is to study the function of the entire hind limb, not just the hind limb bones. The appendicular and axial skeleton of theropods and other tetrapods are segmented lever systems that only make functional sense when soft tissues are included. The CoM, CoP, GRF, and extensor muscle moments are central to understanding locomotion, but are not inherently preserved in the skeleton. Only by deriving these parameters can morphology, motion, and kinetics begin to be reintegrated as they were in life.

FIGURE 4. Comparative analysis of non-avian theropods (open circles) and three birds (filled symbols). A, relative number of mid-stance configurations versus relative GRF magnitude; B, relative maximum vertical GRF versus log body mass; $\mathbf{C}$, relative minimum crouch versus log body mass; Abbreviations: Allo, Allosaurus; Dro, Dromaius; Eud, Eudromia; Gor, Gorgosaurus (juvenile); Str, Struthio; Tyr, Tyrannosaurus; Vel, Velociraptor. 


\section{Tyrannosaurus Stance and Gait}

The 2,391 Tyrannosaurus poses capable of slow running (Fig. 5A, B) are limited to a relatively small region of configuration space. Individual ranges permitted for the hip $\left(19^{\circ}: 50-69^{\circ}\right)$, knee $\left(33^{\circ}: 108-141^{\circ}\right)$ and ankle $\left(46^{\circ}: 132-168^{\circ}\right)$ do not adequately represent our findings, because only one in thirteen poses within this sub-volume has the appropriate coordination among joints. Hip angle was particularly well-resolved by the correct moment sign constraint. If the knee of Tyrannosaurus had a net extensor muscle moment at mid-stance, as in all extant examples of steady-speed bipedal running we could find in the literature, then its flexion/extension axis could not have been far behind the CoM, CoP, and GRF. Simple trigonometry reveals that for a $1.22 \mathrm{~m}$ long thigh and a vertical GRF, the hip angle can be no larger than $71^{\circ}$ if the trunk CoM is $0.4 \mathrm{~m}$ in front of the hip or $55^{\circ}$ if the trunk $\mathrm{CoM}$ is $0.7 \mathrm{~m}$ anterior. Accounting for thigh mass changes these limits by about one degree, but the more vertical femur implied by some reconstructions (Osborn, 1916; Newman, 1970; Tarsitano, 1983) would require the CoM to be at or behind the hip, which is extremely improbable. Likewise, any
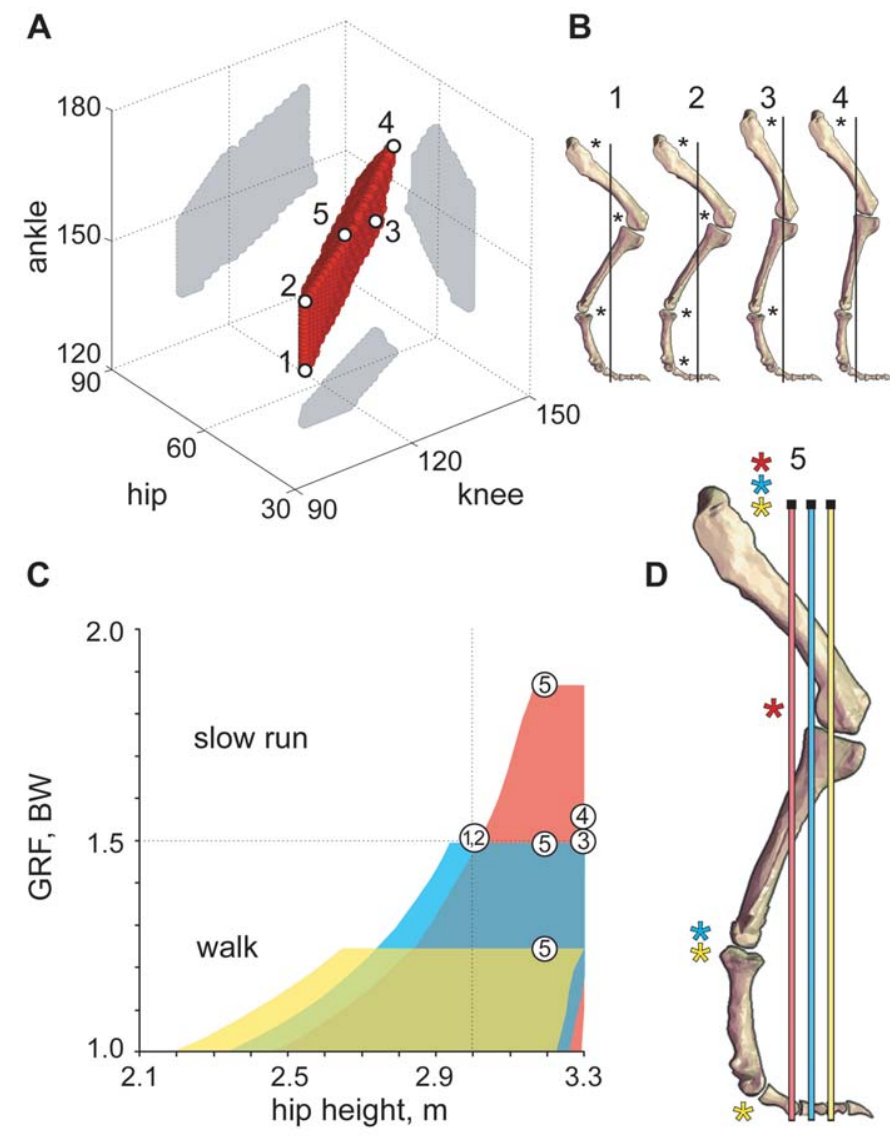

FIGURE 5. Potential mid-stance running poses for Tyrannosaurus. A, slow running poses (maximum GRF 1.5-1.87 BW) are restricted to a narrow wedge of configuration space (red, with grey projections onto angle-angle planes); B, extreme poses: 1 ( $50^{\circ}$ hip, $108^{\circ} \mathrm{knee}, 132^{\circ}$ ankle), $2\left(50^{\circ}, 109^{\circ}, 149^{\circ}\right), 3\left(70^{\circ}, 141^{\circ}, 142^{\circ}\right), 4\left(61^{\circ}, 139^{\circ}, 168^{\circ}\right)$ have low maximum GRFs (circles in C) limited by different "weak" joints (asterisks); C, maximum mid-stance GRFs versus hip height for three trunk CoM locations (red- $0.4 \mathrm{~m}$, blue- $0.5 \mathrm{~m}$, yellow- $0.6 \mathrm{~m}$ anterior to the hip); D, force output of pose $5\left(58^{\circ}, 124^{\circ}, 147^{\circ}\right.$; three circles in $\left.\mathbf{C}\right)$ declines rapidly when the CoM (colored bars) moves forward. Colored asterisks mark "weak" joints for each CoM. Slow running is only feasible when the hip is higher than about $3 \mathrm{~m}$ and the trunk CoM is located closer than $0.5 \mathrm{~m}$ to the hip. mid-stance pose with either the toes or the knee axis significantly posterior to the CoM (e.g., Henderson, 2003) is untenable for steady-speed locomotion, based on our knowledge of living biped kinetics.

The pattern of maximum GRF throughout the viable subset is complex. An intimate relationship between limb configuration and function is revealed by variation in the 'weak' joints with pose (Fig. 5B, D) and the sensitivity of force output to trunk CoM location (Fig. 5C, D). Importantly, GRF values of $1.5 \mathrm{BW}$ and higher could not be achieved in Tyrannosaurus if the trunk CoM was farther than $0.5 \mathrm{~m}$ from the hip (Fig. 5C). For example, pose 5 with hip $58^{\circ}$, knee $124^{\circ}$, and ankle $147^{\circ}$ is consistent with a slow running GRF of $1.87 \mathrm{BW}$ if the trunk CoM was located $0.4 \mathrm{~m}$ anterior to the hip (red, Fig. 5C, D). If the trunk CoM is at $0.5 \mathrm{~m}$, the GRF drops to $1.49 \mathrm{BW}$ (blue) and then to $1.22 \mathrm{BW}$ (yellow) if the trunk CoM is at $0.6 \mathrm{~m}$ (Fig. 4C, D), well below a magnitude needed to run.

The field of dinosaur locomotion has languished in debates about whether tyrannosaur hind limbs were either 'columnar' or 'flexed' (Lambe, 1917; Bakker, 1986; Paul, 1988, 1998; Christiansen, 1999). A configuration space approach more accurately presents reality as a volume of graded hypotheses, rather than a false dichotomy (see also Hutchinson et al., 2005). It has been assumed that a more crouched ('flexed') leg configuration implies greater speeds (Paul, 1988, 1998), but only configurations taller than about $2.97 \mathrm{~m}$ (Fig. 5C), in which all the joint axes stay closer to the GRF vector, are able to mitigate the demands of running on the extensor musculature (e.g., Biewener, 1990). Given that living ostriches crouch $88-90 \%$ at mid-stance (Jindrich et al., 2007; Rubenson et al., 2007), our conservative value of $95 \%$ crouch for Tyrannosaurus may be too lenient, but the degree of relative crouch might scale negatively with size. Dropping maximum hip height to $3.15 \mathrm{~m}(90 \%$ crouch) would further restrict the number of potential running configurations to 951 , the maximum GRF to $1.79 \mathrm{BW}$, and presumably top speed.

As Figure 4C shows, this problem only affects the largest taxon in our analysis. At body masses below $6000 \mathrm{~kg}$, there is no evident adverse scaling of the amount of limb flexion allowed, at least for slow running. Arguing from analogy with smaller living species (e.g., Paul, 1988, 1998) may be of limited value for the largest theropods, as smaller taxa are by definition less affected by adverse scaling.

\section{Validation}

Not surprisingly, constraints based on extant bipeds did not exclude poses actually used at mid-stance by running ostriches and emus. As other running birds are grossly similar and related methods have been checked using additional avian and nonavian species (Hutchinson, 2004a), we are confident that constraint-based exclusion is validated. Extinct theropods could have violated what we consider general constraints by acting outside the bounds of living bipeds, but until solid evidence of such novel mechanics can be found we should conservatively assume functional continuity of these bone-muscle systems.

\section{Scaling}

Constraint-based exclusion reveals the strong influence of body size on a hind limb's ability to produce a vertical force at mid-stance (Fig. 4). Within non-avian taxa, larger theropods show a more rapid decline in the relative number of poses with increasing GRF than smaller theropods (Fig. 4A). For Tyrannosaurus, viable configurations quickly run out as the GRF approaches $2 \mathrm{BW}$, suggesting that top speed and running performance could have been force-limited. Allosaurus, the second largest taxon, also suffers a dramatic reduction in viable poses with increased force, but a GRF of almost $4 \mathrm{BW}$ (sufficient 
for relatively rapid running) is possible given our assumptions (as in Hutchinson, 2004b; Sellers and Manning, 2007). Birds also show a size effect, but their scaling curves are shallower, creating overlap with larger-bodied non-birds. We calculated a maximum GRF for each species by finding the optimum combination of CoM and CoP locations (within our ranges of uncertainty) and pose (Fig. 4B). The steep decline in non-avian theropod performance with size leads to the prediction that an animal much larger than Tyrannosaurus might not be able to produce sufficient force for even slow running.

Although adverse scaling was expected (e.g., Hutchinson and Garcia, 2002), the high maximum GRF values for some taxa are somewhat surprising. The three birds, Velociraptor, and juvenile Gorgosaurus appear to be able to generate vertical forces in excess of $6 \mathrm{BW}$ - far greater than GRFs measured during steady locomotion in non-hopping living animals (reviewed in Hutchinson, 2004a). Clearly, factors other than peak mid-stance muscle force are limiting top running speed in these smaller theropods. These unidentified factors may also explain the anomalously high running speeds calculated for Compsognathus and other small theropods by Sellers and Manning (2007). In light of the large number of options available to most theropods at running GRFs of 2-4 BW, further optimization analysis and a consideration of the entire stride cycle may reveal why specific poses are chosen over so many alternatives.

\section{Alternative Methods}

Over the past decade, computational tools for creating and manipulating 3-D models have given paleobiologists a new way to approach fundamental questions about movement in dinosaurs and other extinct organisms (Hutchinson and Gatesy, 2006). Commercial animation packages or proprietary code allow bones to be articulated by virtual joints into 'digital marionettes,' which can be posed to explore hypothetical positions and motions (Gatesy et al., 1999; Stevens, 2002; Henderson, 2003; Hutchinson et al., 2005; Nicolas et al., 2007). But the power of these computational tools to generate hypotheses creates a new problem-how to choose among countless alternatives?

The mid-stance limb pose of a close living relative or presumed functional analogue could be duplicated, yet animals like Tyrannosaurus are so unlike any living species. A 'realistic' pose could also be selected based on intuition, but such subjectivity should be a last resort. Alternatively, excess degrees of freedom could be reduced by invoking rules of kinematic coordination. For example, the knee and ankle might be required to have the same joint angle (Henderson, 2003) or ratio of angles, thereby confining poses to a plane in our configuration space. However, intralimb coordination is a major goal of reconstruction and should be an output of a model, not an input. A similar decrease in kinematic redundancy comes from restricting the anteroposterior location of the hip with respect to the toes; this is why our vertical GRF constraint is so effective. But the sensitivity of both pose viability and maximum GRF to CoM location (Fig. 5C) raises concerns about oversimplifying mid-stance kinetics and kinematics. Blanco and Jones (2005) calculated tibial stress at mid-stance in order to estimate top speed in phorusrhacids (extinct, flightless birds) assuming a vertical GRF passing through the hip (i.e., a CoM located at the hip). Although such a posterior CoM position might be appropriate for the hind limb of ungulate mammals (Blanco et al., 2003), it is not known in any extant bird (e.g., Roberts, 2001; Jindrich et al., 2007) and likely yields erroneous joint moments and bone loading.

More generally, we are skeptical of any method claiming to produce a single solution (e.g., one speed, pose, stride cycle, etc.), which is likely beyond the resolution of any paleontological analysis. Although a running theropod clearly had a single CoM location at mid-stance of any one stride, we are obligated to treat this critical variable as a range in order to honestly express our uncertainty. We prefer to cope with unknown coordination among joint angles, ranges of possible $\mathrm{CoM}$ and $\mathrm{CoP}$ location, and indefinite hip height to avoid losing sight of the uncertainties involved in paleobiological reconstructions. A configuration space approach offers a way of visualizing the cloud of most probable hypotheses and exploring the interaction of multiple constraints across multiple joints.

Optimization-based simulation approaches (e.g., Anderson and Pandy, 2001; Sellers et al., 2005; Sellers and Manning, 2007) are promising avenues, but depend on accurate identification of optimization criteria, information about many more input parameters, and validation with actual kinematics and kinetics of extant taxa. Our somewhat less ambitious effort to restrict the mid-stance pose could act synergistically as a "filter" against which simulations could be cross-checked or constrained. Regardless, we expect that the future of reconstruction rests in maintaining a high fidelity to biomechanical principles from extant animals, especially when combined with validation and sensitivity analysis.

\section{Future Directions}

Identifying potential mid-stance configurations is a far cry from reconstructing entire stride cycles for Tyrannosaurus or reconstructing locomotor evolution, but we consider this an important starting point. The relationship between GRF magnitude and speed in living animals (e.g., Alexander et al., 1979; Arampatzis, Brüggemann, and Metzler, 1999; McMahon and Cheng, 1990; Robilliard and Wilson, 2006) may permit estimation of maximum sustained running speed from mid-stance. Yet a top speed value without strong kinematic and kinetic validation may be premature. From our solution set, we should be able to move backward in time to reconstruct potential pose sequences for the first half of the stance phase. At the moment of limb touchdown, hip height will be greater than at mid-stance and the outstretched limb will be longer, thereby decreasing the number of possible poses. Reconstructing the second half of stance will be more difficult because lifting the phalanges adds additional joints to an already redundant system, but toes cannot simply be ignored.

We are actively seeking geometric and kinetic constraints for the swing phase, as well as additional constraints for stance. To more accurately model muscle-tendon interactions, the addition of tendon properties into the model would improve resolution (Sellers and Manning, 2007), but issues such as slack length make this far from straightforward. Although we doubt that single values for tendon properties, trunk orientation, phalangeal position, digital pads, joint ranges of motion, extensor muscle mass, fascicle length, and CoM position will ever be defensible, this study lays crucial groundwork for detailed sensitivity analysis of all our assumptions and their interactions.

Given the power of modern hardware and software, the question is no longer how can we make extinct animals move, but how should we make them move? Constraint-based exclusion is a promising method of evaluating hypothetical poses in an extinct animal without high computational demands. It could be used to examine locomotion in other bipedal diapsids and mammals (primates, marsupials, rodents, giant sloths). Modification and addition of constraints should allow analysis of quadrupeds as well. We see no reason why constraints governing motion of limbs and other appendages in swimming, flight, feeding, and other behaviors could not be identified for pose exclusion. A unique benefit of this approach is its transparency and repeatability; just as systematists can analyze a published character matrix, other workers could reproduce our findings and evaluate the effects of modifying our assumptions and constraints. 
We hope that our case study will stimulate paleobiologists to find new ways to move the field toward more objectivity and lucidity.

\section{ACKNOWLEDGMENTS}

Thanks to Tom Roberts, Vivian Allen, Lei Ren, Charlotte Miller, Jonas Rubenson, Beth Brainerd and other members of the Brown Morphology Group and Structure and Motion Laboratory. We are grateful to several reviewers for critical comments on earlier versions of this manuscript. We appreciate software support from Autodesk (S.M.G.). Funding was provided by the National Science Foundation (S.M.G.), a Bushnell Faculty Research Grant (S.M.G.), the BBSRC (J.R.H.) and the Department of Veterinary Basic Sciences at The Royal Veterinary College (J.R.H.).

\section{LITERATURE CITED}

Alexander, R. McN. 2004. Principles of Animal Locomotion. Princeton University Press, Princeton, New Jersey, 371 pp.

Alexander, R. McN., G. M. O. Maloiy, R. Njau, and A. S. Jayes. 1979 Mechanics of running of the ostrich (Struthio camelus). Journal of Zoology, London 187:169-178.

Anderson, F. C., and M. G. Pandy. 2001. Dynamic optimization of human walking. Journal of Biomechanical Engineering 123:381-390.

Arampatzis, A., G. P. Brüggemann, and V. Metzler. 1999. The effect of speed on leg stiffness and joint kinetics in human running. Journal of Biomechanics 32:1349-1353.

Bakker, R. T. 1986. Dinosaur Heresies. William Morrow, New York, 481 pp.

Biewener, A. A. 1990. Biomechanics of mammalian terrestrial locomotion. Science 250:1097-1103.

Biewener, A. A., R. McN. Alexander, and N. C. Heglund. 1981. Elastic energy storage in the hopping of kangaroo rats (Dipodomys spectabilis). Journal of Zoology, London 195:369-383.

Biewener, A. A., C. T. Farley, T. J. Roberts, and M. Temaner. 2004. Muscle mechanical advantage of human walking and running: implications for energy cost. Journal of Applied Physiology 97:2266-2274.

Blanco, R. E., and W. J. Jones. 2005. Terror birds on the run: a mechanical model to estimate its maximum running speed. Proceedings of the Royal Society, B. 272:1769-1773.

Blanco, R. E., R. Gambini, and R. A. Fariña. 2003. Mechanical model for theoretical determination of maximum running speed in mammals. Journal of Theoretical Biology 222:117-125.

Carpenter, K. 2002. Forelimb biomechanics of nonavian theropod dinosaurs in predation. Senckenbergiana Lethaea 82:59-76.

Cavagna, G. A., N. C. Heglund, and C. R. Taylor. 1977. Mechanical work in terrestrial locomotion: two basic mechanisms for minimizing energy expenditure. American Journal of Physiology 233:R243-R261.

Christiansen, P. 1999. Long bone scaling and limb posture in non-avian theropods: evidence for differential allometry. Journal of Vertebrate Paleontology 19:666-680.

Farley, C. T., and D. P. Ferris. 1998. Biomechanics of walking and running: center of mass movements to muscle action. Exercise and Sport Sciences Reviews 26:253-286.

Farlow, J. O., M. B. Smith, and J. M. Robinson. 1995. Body mass, bone "strength indicator," and cursorial potential of Tyrannosaurus rex. Journal of Vertebrate Paleontology 15:713-725.

Farlow, J. O., S. M. Gatesy, T. R. Holtz, J. R. Hutchinson, and J. M. Robinson. 2000. Theropod locomotion. American Zoologist 40:640-663.

Gatesy, S. M. 1990. Caudofemoral musculature and the evolution of theropod locomotion. Paleobiology 16:170-186.

Gatesy, S. M. 1999. Guineafowl hind limb function I: cineradiographic analysis and speed effects. Journal of Morphology 240:127-142.

Gatesy, S. M., K. M. Middleton, F. A. Jenkins, Jr. and N. H. Shubin. 1999. Three-dimensional preservation of foot movements in Triassic theropod dinosaurs. Nature 399:141-144.

Gishlick, A. D. 2001. The function of the manus and forelimb of Deinonychus antirrhopus and its importance for the origin of avian flight; pp. 301-318 in J. Gauthier and J. F. Gall (eds.), New Perspectives on the Origin and Early Evolution of Birds. Peabody Museum of Natural History, New Haven, Connecticut.
Günther, M., and R. Blickhan. 2002. Joint stiffness of the ankle and the knee in running. Journal of Biomechanics 35:1459-1474.

Hallemans, A., K. D'Aout, D. de Clerq, and P. Aerts. 2003. Pressure distribution patterns under the feet of new walkers: the first two months of independent walking. Foot and Ankle International 24:444-453.

Henderson, D. 2003. Footprints, trackways, and hip heights of bipedal dinosaurs-testing hip height predictions with computer models. Ichnos 10:99-114.

Herr, H., and M. Popovic. 2008. Angular momentum in human walking. Journal of Experimental Biology 211:467-481.

Horner, J. R., and D. Lessem. 1993. The Complete T. rex. Simon and Schuster, New York, 239 pp.

Hutchinson, J. R. 2004a. Biomechanical modeling and sensitivity analysis of bipedal running ability I. extant taxa. Journal of Morphology 262:421-440.

Hutchinson, J. R. 2004b. Biomechanical modeling and sensitivity analysis of bipedal running ability II. extinct taxa. Journal of Morphology 262:441-461.

Hutchinson, J. R., and M. Garcia. 2002. Tyrannosaurus was not a fast runner. Nature 415:1018-1021.

Hutchinson, J. R., and S. M. Gatesy. 2006. Dinosaur locomotion: beyond the bones. Nature 440:292-294.

Hutchinson, J. R., V. Ng-Thow-Hing, and F. C. Anderson. 2007. A 3D interactive method for estimating body segmental parameters in animals: application to the turning and running performance of $T y$ rannosaurus rex. Journal of Theoretical Biology 246:660-680.

Hutchinson, J. R., F. C. Anderson, S. S. Blemker, and S. L. Delp. 2005. analysis of hindlimb muscle moment arms in Tyrannosaurus rex using a three-dimensional musculoskeletal computer model: implications for stance, gait, and speed. Paleobiology 31:676-701.

Jindrich, D. 1., N. C. Smith, K. Jespers, and A. M. Wilson. 2007. Mechanics of cutting maneuvers by ostriches (Struthio camelus). Journal of Experimental Biology 210:1378-1390.

Lambe, L. M. 1917. The Cretaceous carnivorous dinosaur Gorgosaurus. Geological Survey of Canada Memoir 100:1-84.

Main, R. P., and A. A. Biewener. 2007. Skeletal strain patterns and growth in the emu hindlimb during ontogeny. Journal of Experimental Biology 210:2676-2690.

McGowan, C. P., R. V. Baudinette, and A. A. Biewener. 2005. Joint work and power associated with acceleration and deceleration in tammar wallabies (Macropus eugenii). Journal of Experimental Biology 208:41-53.

McMahon, T. A., and G. C. Cheng. 1990. The mechanics of running: how does stiffness couple with speed. Journal of Biomechanics 23:65-78.

Medler, S. 2002. Comparative trends in shortening velocity and force production in skeletal muscle. American Journal of Physiology. Regulatory, Integrative and Comparative Physiology 283: R368-R378.

Mendez, J., and A. Keys. 1960. Density and composition of mammalian muscle. Metabolism 9:184-188.

Middleton, K. M. 2003. Morphology, evolution, and function of the avian hallux. Ph.D. thesis, Brown University, Providence, Rhode Island.

Munro, C. F., D. I. Miller, and A. J. Fuglevand. 1987. Ground reaction forces in running: a reexamination. Journal of Biomechanics 20:147-155.

Newman, B. H. 1970. Stance and gait in the flesh-eating dinosaur Tyrannosaurus. Biological Journal of the Linnean Society 2:119-123.

Nicolas, G., F. Multon, G. Berillon, and F. Marchal. 2007. From bone to plausible bipedal locomotion using inverse kinematics. Journal of Biomechanics 40:1048-1057.

Osborn, H. F. 1916. Skeletal adaptations of Ornitholestes, Struthiomimus and Tyrannosaurus. Bulletin of the American Museum of Natural History 35:733-771.

Paul, G. S. 1988. Predatory Dinosaurs of the World. Simon and Schuster, New York, 464 pp.

Paul, G. S. 1998. Limb design, function and running performance in ostrich-mimics and tyrannosaurs. Gaia 15:257-270.

Roberts, T. J. 2001. Muscle force and stress during running in dogs and wild turkeys. Bulletin of the Museum of Comparative Zoology 156:283-295.

Roberts, T. J. 2002. The integrated function of muscles and tendons during locomotion. Comparative Biochemistry and Physiology A 133:1087-1099. 
Roberts, T. J., R. L. Marsh, P. G. Weyand, and C. R. Taylor. 1997. Muscular force in running turkeys: the economy of minimizing work. Science 275:1113-1115.

Roberts, T. J., and J. A. Scales. 2002. Mechanical power output during running accelerations in wild turkeys. Journal of Experimental Biology 205:1485-1494.

Robilliard, J. J., and A. M. Wilson. 2006. Prediction of kinetics and kinematics of running animals using an analytical approximation to the planar spring-mass system. Journal of Experimental Biology 208:4377-4389.

Rubenson, J., D. B. Heliams, D. G. Lloyd, and P. A. Fournier. 2003. Gait selection in the ostrich: mechanical and metabolic characteristics of walking and running with and without an aerial phase. Proceedings of the Royal Society, B. 271:1091-1099.

Rubenson, J., D. G. Lloyd, T. F. Besier, D. B. Heliams, and P. A. Fournier. 2007. Running in ostriches (Struthio camelus): three-dimensional joint axes alignment and joint kinematics. Journal of Experimental Biology 210:2548-2562.

Sellers, B. I., and P. L. Manning. 2007. Estimating dinosaur maximum running speeds using evolutionary robotics. Proceedings of the Royal Society, B. 274:2711-2716.
Sellers, W. I., G. M. Cain, W. Wang, and R. H. Crompton. 2005. Stride lengths, speed and energy costs in walking of Australopithecus afarensis: using evolutionary robotics to predict locomotion of early human ancestors. Journal of the Royal Society Interface 2:431-441.

Senter, P. 2005. Function in the stunted forelimbs of Mononykus olecranus (Theropoda), a dinosaurian anteater. Paleobiology 31:373-381.

Stevens, K. A. 2002. DinoMorph: parametric modeling of skeletal structures. Senckenbergiana Lethaea 82:23-34.

Tarsitano, S. 1983. Stance and gait in theropod dinosaurs. Acta Palaeontologica Polonica 28:251-264.

Vereecke, E., K. D'Aout, D. de Clerq, L. Van Elsacker, and P. Aerts. 2003. Dynamic plantar pressure distribution during terrestrial locomotion of bonobos (Pan paniscus). American Journal of Physical Anthropology 120:373-383.

Vereecke, E., K. D'Aout, L. Van Elsacker, D. de Clerq, and P. Aerts 2005. Functional analysis of the gibbon foot during terrestrial bipedal walking: plantar pressure distributions and three-dimensional ground reaction forces. American Journal of Physical Anthropology 128:659-669.

Submitted April 10, 2008; accepted August 17, 2008. 